\title{
Nasal Injury with Continuous Positive Airway Pressure: Need for "Privileging" Nursing Staff
}

\author{
Nihaz Naha ${ }^{1} \cdot$ Femitha Pournami $^{1}$ (D) Jyothi Prabhakar ${ }^{1} \cdot$ Naveen Jain $^{1}$
}

Received: 3 August 2018 / Accepted: 9 April 2019 / Published online: 24 April 2019

(C) Dr. K C Chaudhuri Foundation 2019

\begin{abstract}
Objectives Use of continuous positive airway pressure (CPAP) in neonates is associated with nasal injury (NI) for which various risk factors related to the neonatal characteristics and properties of interfaces used have been reported. "Privileging" of nursing staff may influence safety and incidence of adverse events. In this prospective cohort study, authors studied the incidence of NI and risk factors for NI in babies requiring CPAP after privileging staff for CPAP care bundles.

Methods All neonates on CPAP over a 6-mo period were included. Standard operating procedures were formulated and staff of NICU (nurses and doctors) were educated at the start of the study and periodically in 6 comprehensive areas of careencompassing position of head, prongs and cap; nasal suctioning and interruptions in pressure on the nose. The staff who completed the training and evaluation were declared as "privileged". NI (measured by a standard staging) and risk factors were predefined and studied.

Results Of the 51 babies who required respiratory supports, 35 required CPAP care. Nine babies (25\%) out of 35 who required CPAP had NI (2, 4, 3 babies had stages 1, II and III of NI respectively). Seventy seven percent of babies were cared for by privileged nurses. NI was significantly higher when cared for by non-privileged staff ( $66 \% v$ s. $11 \%$, unadjusted RR $=6.75,95 \%$ CI 2.16 21.09). All other risk factors were not significant.

Conclusions NI was noted in 25\% neonates on CPAP, and those cared for by non-privileged staff had higher chances of NI. Quality processes and emphasis on continued monitoring and evaluation of nursing skills may help prevent these untoward complications.
\end{abstract}

Keywords CPAP $\cdot$ Nasal injury $\cdot$ Privileging $\cdot$ Care bundles

\section{Introduction}

Extensive and early use of non-invasive respiratory supports in neonatal intensive care units (NICU) has markedly reduced the need for endotracheal intubation and ventilation. Nonetheless, the use of this technology is associated with nasal injuries (NI) [1]. This is an adverse event with potential long and short-term consequences. Although many risk factors for NI have previously been analyzed- gestational age, duration of continuous positive airway pressure (CPAP), type of interface, method of fixation, use of protective dressings; authors believe that the technique of application and nursing

Femitha Pournami

femi_shifas@yahoo.com

1 Department of Neonatology, Kerala Institute of Medical Sciences, Trivandrum, Kerala 695029, India care during CPAP are fundamental determinants of NI $[2,3]$. There is a dearth of literature which systematically looks at effect of a structured nursing training on quality of care as measured by rates of adverse events.

This prospective cohort study aimed to analyze incidence of NI and identify risk factors for NI in authors' unit after privileging the staff for standard CPAP care bundles.

\section{Material and Methods}

This study was conducted over 6 mo from June through December 2015 in a 26 bedded NNF Level 3B accredited NICU of a tertiary referral hospital in South India. CPAP is administered by Hudson short binasal prongs of appropriate recommended sizes, either by bubble CPAP via Fisher and Paykel®stand-alone equipment; or by CPAP mode of Dragger®Babylog 8000plus. All are equipped with Fisher 
and Paykel humidifier system with auto fill chamber and disposable ventilator tubings with double heated wire.

Standard operating procedures were formulated. Key points in the CPAP bundle included the following components: 1 . Appropriate position of infant's head with blanket and neck rolls to prevent excessive movements of interface; 2 . Appropriate hat size and position; 3. Prongs position, where prongs did not pull on nose even with movement of head; 4 . Gentle nasal suction; 5 . Routine nasal massage; 6 . Blow by breaks where the interface was removed from the nostrils at least twice every nursing shift. If the baby was on $\mathrm{FiO}_{2}>$ 0.21 , the nursing staff held free flow $\mathrm{O}_{2}$ around $2 \mathrm{~cm}$ from the nostril. The baby was under continuous pulse oximetry monitoring during this period. The average duration of each break was $30 \mathrm{~min}$.

The staff nurses of NICU were educated through AprilMay 2015 in batches as 5 sessions describing each particular component of CPAP care. Classes included face to face discussions, audiovisual aids and hands on demonstrations of each component described above, on mannequins. Staff were to attend at least one class about each component of the care bundle; after which they were observed for compliance on the first subsequent baby who was allocated to them. Senior privileged nurses and senior residents supervised the respective nurses for compliance and level of expertise. If unsatisfactory, the areas of deficiencies were identified. Re-education with more audio-visual and hands on demonstrations were conducted to address these. Members with lowest compliance were allotted special sessions. Nursing staff were certified as "privileged" after all these sessions and steps were completed and they were issued a "privileging form of completion". Repeat sessions were continued throughout the study fortnightly. The principal investigator along with the in-charge nurses of NICU conducted daily audit of compliance to CPAP bundle check list and identified gaps.

NI was classified according to the National Pressure Ulcer Advisory Panel (NPUAP) and European Pressure Ulcer Advisory Panel (EPUAP) [4]; Stage 1: Intact skin with non-blanchable redness, Stage 2: Presence of ulcer or superficial erosion with partial skin loss, Stage III: Presence of necrosis and full skin loss. Examinations of the nose were done by the allocated nurse and team leader (in-charge nurse of each shift) and senior resident on call. Nasal injury, if present were confirmed and staged by the Principal investigator and consultant. Once NI was noted, change in mode of respiratory therapy and treatment of NI was done as per the unit's protocol according to the staging.

Birth weight, gestational age, CPAP hours, care by privileged/ non-privileged staff were recorded. Only one kind of nasal interface is used at authors' institution as the unit is most comfortable and experienced in the use of Hudson prongs.
If more than $95 \%$ of the nursing shifts while the baby was on respiratory support were covered by "privileged" staff, then that baby was considered to be cared by privileged staff. Percentage of shifts covered by privileged staff was defined as (number of shifts where baby was cared by privileged staff while on CPAP) $\div$ (number of shifts that the baby was on CPAP) $\times 100$. There were circumstances when allocation could not always be privileged personnel during staff shortage.

The authors planned to enroll all eligible consecutive babies over 6 mo period.

\section{Results}

Over the six month study period, 51 babies required respiratory support, $16(31.4 \%)$ ventilator care alone, $22(43.1 \%)$ CPAP care alone and 13 (25.5\%) required both.

Twenty-four out of 31 nurses posted in the NICU during the study period were certified as "privileged" for CPAP care before the first baby was enrolled in June 2015. Through the study period, more number of nurses could complete the sessions and demonstrated compliance to the CPAP bundle components. Six hundred sixty eight nursing shifts of ventilator/CPAP babies were audited. Seventy-seven percent of babies were cared for by privileged nurses. Nine babies $(25 \%)$ out of 35 babies who required CPAP had NI (2, 4, 3 babies had stages 1,2 and 3 of NI respectively).

Risk factors analysis revealed no significant association of birth weight $(p=0.22)$, gestational age $(p=0.45)$ and duration of CPAP $(p=0.89)$ with nasal injury (Table 1$)$. There was a significantly higher risk of nasal injury in those cared for by non- privileged staff ( $66 \%$ vs. $11 \%$, unadjusted $\mathrm{RR}=6.75$, 95\% CI 2.16-21.09).

Table 1 Baseline characteristics of babies with and without nasal injury $(n=35)$

\begin{tabular}{lllll}
\hline $\begin{array}{l}\text { Sl. } \\
\text { no. }\end{array}$ & Characteristic & $\begin{array}{l}\text { No nasal injury } \\
(n=26)\end{array}$ & $\begin{array}{l}\text { Nasal injury } \\
(n=9)\end{array}$ & $P$ value \\
\hline 1 & $\begin{array}{c}\text { Birth weight } \\
\text { in grams* } \\
\text { Gestational age } \\
\text { in weeks* }\end{array}$ & $\begin{array}{c}1493 \\
(945-2210)\end{array}$ & $\begin{array}{c}1385 \\
(883-1797)\end{array}$ & 0.22 \\
2 & $22(84-34)$ & $31(28-33)$ & 0.45 \\
3 & $\begin{array}{c}\text { Inborn** } \\
\text { Antenatal steroids } \\
\text { covered ** }\end{array}$ & $10(38.5)$ & $8(88.9)$ & 0.98 \\
5 & $\begin{array}{c}\text { Cesarean } \\
\text { delivery** }\end{array}$ & $18(69.2)$ & $8(88.9)$ & 0.14 \\
6 & $\begin{array}{c}\text { Hours of CPAP } \\
\text { support* }\end{array}$ & $47(11-99)$ & $48(13-187)$ & 0.26 \\
\hline
\end{tabular}

*Median (25-75 quartiles), **n (\%) 


\section{Discussion}

With increasing survival and better outcomes of neonates at lower gestations, there comes a paradigm shift of priorities to improved quality of care. The role of trained nurses in neonatal intensive care cannot be overemphasized. Health care staff are selected primarily based on their educational qualifications. Credentialing is an essential process that healthcare organizations and practices perform to ensure those providing services are qualified to do so. A more imperative need is the further process of "privileging," whereby a professional is authorized to provide a specific scope of patient care services. Privileging is performed in conjunction with evaluation of an individual's clinical qualifications along with performance in that area of care [5]. Literature supports the fact that NICUs across the world do not meet standard staffing recommendations; and that nurse-to-patient ratios appear to affect outcomes [6]. There is a dearth of publications evaluating the effect of having a structured on-going education, monitoring and evaluation plan for each skill of every staff. These, in turn, may contribute to improved safety and quality of health care services.

The authors conducted a prospective cohort study to evaluate the incidence of NI during CPAP supports after privileging the NICU staff in CPAP care bundles. They found an incidence of $25 \% \mathrm{NI}$ in their cohort. Nasal trauma is a welldocumented complication of non-invasive respiratory support [7]. The local pressure of nasal prongs can cause NI in the newborn due to the cutaneous vulnerability [8]. These injuries can disfigure the infant, sometimes, permanently. In the present audit, nasal injury was seen in $25 \%$ babies, with more incidence of stage $2(44 \%)$ and stage 3(33\%) injuries. In a study conducted by Fischer et al., $(n=989) 42.5 \%$ neonates developed nasal trauma and there were $88.3 \%$ of stage I, $11 \%$ of stage II and $0.7 \%$ of stage III nasal trauma [9].

The risk factors - birth weight, gestational age, duration of CPAP and nursing care characteristics were analyzed. The other previously studied factors like type of interface, method of fixation, use of lubricants and humidification were the same in all babies throughout the period of study. Besides anatomical factors, such as end-vascularization of the columella and nostrils, Diblasi, emphasized that fixation technique was also an important factor. The lack of stabilization and, hence, excessive movement of the prongs could result in nasal injury [10]. Diligence in ensuring the appropriate positioning of prongs relative to nose and frequent repositioning is necessary [11]. Previous studies report that duration of CPAP is a significant risk factor associated with development of nasal injury, after birth weight, gestational age, and nasal device used had been controlled [12]. The prolonged use of CPAP resulted in more pressure, and if there is any area of pressure points exerted by the device, this would definitely cause trauma, no matter what kind of mode. However, injury to the columella in CPAP had been reported as early as 3 days after CPAP [11]. In the present study the days on CPAP were not associated with increased incidence of nasal injury. Günlemez et al. demonstrated that the use of silicone gel on the surface of the nostrils reduced the incidence of nasal injury in preterm infants [13]. Collins et al. in a study comparing nasal trauma in CPAP vs. Heated humidified high flow nasal cannula found that, the use of protective dressing was not associated with decreased nasal trauma for infants on CPAP [14]. Hydrocolloid shields have been devised to protect skin, and initially showed encouraging results [15]. However, pressure is often the problem, rather than friction. Once the skin barrier has been injured, the use of these products may promote further breakdown. A checklist with all the steps necessary to prevent complications for patients on CPAP has been useful in some NICUs to improve the consistency of care. In the present study, NI rates were significantly less in babies cared by privileged staffs (11\%) compared to babies cared by non-privileged staff $(75 \%)$. The authors feel that the low incidence of NI in authors' unit itself may be due to the privileging process, which made the staff more alert on the care of baby on CPAP.

The authors admit that the study population includes a small number of babies. Although they made maximal efforts to measure the percentage of shifts covered by privileged/nonprivileged staff, it is true that during busy days, senior staff did contribute to care of all babies and strict allocation with no cross-over could not be assured.

Although it seems obvious that nursing care quality would affect outcomes, this study aids in demonstrating an objective measure. Of all the risk factors analysed, care by privileged nurses independently and significantly decreased the chances of nasal injury during CPAP. This may help in advocacy for continued nursing training and evaluation of their performances. Hospital policies differ with some even insisting on staff rotations through various specialities. These can affect number of trained staff available for a highly demanding and skill dependent area like neonatal patient care. Privileging existing staff nurses and empowering them to deliver quality care can go a long way in reducing complications related to therapy.

\section{Conclusions}

In the present study, $25 \%$ incidence of NI was noted in babies on CPAP; $8 \%$ had higher stages of NI. There was a significantly lower rate of $\mathrm{NI}$ in babies cared by nurses privileged for CPAP.

Acknowledgements The authors acknowledge the contributions of Senior nurses of the Neonatal Intensive Care Unit- Ms. Sandhya S V, Ms. Anupama A in supervising the privileging process.

Authors' Contribution NJ conceived the study; NN, FP planned the study and wrote the protocol; NN, JP and FP did the privileging process; NN did data collection; NN, FP, JP, NJ did data analysis; NN, FP drafted the manuscript; $\mathrm{NJ}$ finalised the manuscript and is guarantor for this article. 


\section{Compliance with Ethical Standards}

Conflict of Interest None.

\section{References}

1. Jatana KR, Oplatek A, Stein M, Phillips G, Kang DR, Elmaraghy CA. Effects of nasal continuous positive airway pressure and canula use in the neonatal intensive care setting. Arch Otolaryngol Head Neck Surg. 2010;136:287-91.

2. Ahmad SM, Bhat AA, Akhthar R, Wani MA, Wani T. Nasal trauma in neonates receiving CPAP and its prevention in tertiary care hospital. J Med Sci. 2017;20:96-100.

3. Chen C-Y, Chou A-K, Chen Y-L, Chou H-C, Tsao P-N, Hsieh W-S. Quality improvement of nasal continuous positive pressure therapy in neonatal intensive care unit. Pediatr Neonatol. 2017;58:229-35.

4. Black J, Baharestani MM, Cuddigan J, et al; National Pressure Ulcer Advisory Panel's updated pressure ulcer staging system. National Pressure Ulcer Advisory Panel's updated pressure ulcer staging system. Adv Skin Wound Care. 2007;20:269-74.

5. NAPNAP Position Statement. Position statement on credentialing and privileging for nurse practitioners. J Pediatr Health Care. 2016;30:A20-1.

6. Watson SI, Arumlampalam W, Petrou S, et al. The effects of a one-to-one nurse-to-patient ratio on the mortality rate in neonatal intensive care: a retrospective longitudinal population based study. Arch Dis Child Fetal Neonatal Ed. 2016;101:F195-200.

7. Robertson NJ, McCarthy LS, Hamilton PA, Moss AL. Nasal deformities resulting from flow driver continuous positive airway pressure. Arch Dis Child Fetal Neonatal Ed. 1996;75:F209-12.
8. Cartlidge P. The epidermal barrier. Semin Neonatol. 2000;5: 273-80.

9. Fischer C, Bertelle V, Hohlfeld J, Forcada-Guex M, StadelmannDiaw C, Tolsa JF. Nasal trauma due to continuous positive airway pressure in neonates. Arch Dis Child Fetal Neonatal Ed. 2010;95: F447-51.

10. Diblasi RM. Nasal continuous positive airway pressure (CPAP) for the respiratory care of the newborn infant. Respir Care. 2009;54: 1209-35.

11. McCoskey L. Nursing care guidelines for prevention of nasal breakdown in neonates receiving nasal continuous positive airway pressure. Adv Neonatal Care. 2008;8:116-24.

12. Yong S-C, Chen S-J, Boo N-Y. Incidence of nasal trauma associated with nasal prong versus nasal mask during continuous positive airway pressure treatment in very low birthweight infants: a randomized control study. Arch Dis Child Fetal Neonatal Ed. 2005;90:F480-3.

13. Günlemez A, Isken T, Gökalp AS, Türker G, Arisoy EA. Effect of silicon gel sheeting in nasal injury associated with nasal CPAP in preterm infants. Indian Pediatr. 2010;47:2657.

14. Collins CL, Barfield C, Horne RS, Davis PG. A comparison of nasal trauma in infants extubated either heated humidified high flow nasal cannulae or nasal continuous positive airway pressure. Eur J Pediatr. 2014;173:181-6.

15. Xie LH. Hydrocolloid dressing in preventing nasal trauma secondary to nasal continuous positive airway pressure in preterm infants. World J Emerg Med. 2014;5:218-22.

Publisher's Note Springer Nature remains neutral with regard to jurisdictional claims in published maps and institutional affiliations. 